\title{
ON INTERPOLATION TO A GIVEN ANALYTIC FUNCTION BY ANALYTIC FUNCTIONS OF MINIMUM NORM
}

\author{
BY \\ J. P. EVANS AND J. L. WALSH
}

We shall consider here the following problem. Let the region $R_{1}$ of the $z-$ plane contain the points

$$
\begin{aligned}
& \beta_{11}, \\
& \beta_{21}, \quad \beta_{22}, \\
& \beta_{31}, \quad \beta_{32}, \quad \beta_{33},
\end{aligned}
$$

and let the function $f(z)$ be analytic in these points. To study the convergence to $f(z)$ of the sequence of functions $g_{n}(z)$; here $g_{n}(z)$ is analytic throughout $R_{1}$, coincides with $f(z)$ in the points $\beta_{n 1}, \beta_{n 2}, \cdots, \beta_{n n}$, and among all functions with these two properties has the least norm in $R_{1}$. This problem has been previously studied $[6 ; 7]$ where norm is [lub $\left|g_{n}(z)\right|, z$ in $R_{1}$ ], and is now to be studied (\$1) where norm is measured by a surface integral over $R_{1}$, or $(\$ 2)$ a parametric integral over the boundary of $R_{1}$, or (\$3) a line integral over the boundary of $R_{1}$. If the norm is measured by the integral of the square of the modulus, we obtain by this method an expansion of $f(z)$ in a series of orthogonal functions, an expansion whose convergence properties we study ( $\$ 4$ ) in some detail. The asymptotic behavior of these orthogonal functions themselves and of their zeros is investigated in $\$ 5$.

1. Interpolation by functions of minimum norm, surface integrals. If $R_{1}$ is a given region, we define $\mathcal{L}^{q}\left(R_{1}\right)(0<q<\infty)$ as the class of functions $F(z)$ each analytic in $R_{1}$ with $\iint_{R_{1}}|F(z)|^{a} d S<\infty$, and define $\mathcal{L}^{\infty}\left(R_{1}\right)$ as the class of functions $F(z)$ each analytic and bounded in $R_{1}$; otherwise expressed, the norm of $F(z)$ in $R_{1}$ in these respective cases is $\left[\iint_{R_{1}}|F(z)|^{q} d S\right]^{1 / q}$ and its limit $(q \rightarrow \infty)$ lub $\left[|F(z)|, z\right.$ in $\left.R_{1}\right]$. We define $\mathcal{L}_{n}^{a}\left(R_{1}\right)$ as the subclass of $\mathcal{L}^{q}\left(R_{1}\right)$ consisting of those functions of $\mathcal{L}^{q}\left(R_{1}\right)$ which coincide with the given $f(z)$ in the points $\beta_{n 1}, \beta_{n 2}, \cdots, \beta_{n n}$. The functions of class $\mathcal{L}_{n}^{a}\left(R_{1}\right)$ form a normal family in $R_{1}$, and standard methods show that there exists at least one such function $F_{n}(z)$ of minimum norm. The function $F_{n}(z)$ is unique if $1<q<\infty$, and also if $q=\infty$ and $R_{1}$ is simply connected.

If $S$ is any point set, we denote its closure by $\bar{S}$. With the generic notation

$$
V_{\sigma}^{q}(F)=\left[\iint_{R_{\sigma}}|F(z)| q d S\right]^{1 / q},
$$

Received by the editors June 15, 1954. 
where $R_{\sigma}$ is to be defined, our main result can now be formulated:

THEOREM 1.1. Let $R_{1}$ be a finite region whose boundary $C_{1}$ consists of a finite number of mutually disjoint Jordan curves. Let $R_{0}$ be a point set whose boundary $C_{0}$ consists of a finite number of mutually disjoint Jordan curves, such that $\bar{R}_{0}$ lies in $R_{1}$ and separates no point of $R_{1}-\bar{R}_{0}$ from $C_{1}$. Suppose the points (0.1) not necessarily distinct lie in $\bar{R}_{0}$, and that

$$
\lim _{n \rightarrow \infty}\left|\left(z-\beta_{n 1}\right)\left(z-\beta_{n 2}\right) \cdots\left(z-\beta_{n n}\right)\right|^{1 / n}=e^{V_{1}(z)}
$$

uniformly on any closed bounded set in the complement of $\bar{R}_{0}$. Let $V_{2}(z)$ be the function harmonic in $R_{1}$, continuous in $\bar{R}_{1}$, equal to $V_{1}(z)$ on $C_{1}$. Suppose the function $V(z) \equiv V_{1}(z)-V_{2}(z)$ is continuous and equal to $\gamma(<0)$ on $C_{0}$. With the notation $\phi(z) \equiv 1-V(z) / \gamma$, we denote generically by $C_{\sigma}$ the locus $\phi(z)=\sigma$, $0 \leqq \sigma \leqq 1$, in $\bar{R}_{1}-R_{0}$, and by $R_{\sigma}$ the point set consisting of $\bar{R}_{0}$ plus those points of $R_{1}-\bar{R}_{0}$ for which $0<\phi(z)<\sigma$.

Let the given function $f(z)$ be analytic throughout $R_{\rho}$ but not throughout any $R_{\rho^{\prime}}, 0<\rho<\rho^{\prime}<1$. Then for fixed $q, 0<q \leqq \infty$, the sequence of extremal functions $F_{n}(z)$ of class $\mathcal{L}_{n}^{q}\left(R_{1}\right)$ converges to $f(z)$ throughout $R_{p}$, uniformly on any closed subset of $R_{\rho}$, and we have for $0<t \leqq \infty$

$$
\limsup _{n \rightarrow \infty}\left[N_{\sigma}^{t}\left(f-F_{n}\right)\right]^{1 / n}=e^{\gamma(\rho-\sigma)}, \quad 0 \leqq \sigma<\rho ;
$$

we also have for $0<t \leqq \infty$ if $\rho \leqq \sigma<1$ and for $0<t \leqq q$ if $\sigma=1$

$$
\limsup _{n \rightarrow \infty}\left[N_{\sigma}^{t}\left(F_{n}\right)\right]^{1 / n}=e^{\gamma(\rho-\sigma)} .
$$

For $q=\infty$, Theorem 1.1 has already been established (loc. cit.) for $t=\infty$, and follows at once for $0<t<\infty$. We henceforth denote the extremal functions $F_{n}(z)$ for $q=\infty$ by $f_{n}(z)$, and shall employ the latter as comparison functions in our proof. We shall also use the following:

Lemma 1.1. Let $R_{0}$ and $R_{1}$ satisfy the conditions of Theorem 1.1, let $F(z)$ be an arbitrary function of class $\mathcal{L}^{q}\left(R_{1}\right), 0<q \leqq \infty$, and let $T$ be any closed set interior to $R_{\sigma}, 0 \leqq \sigma \leqq 1$. Then we have

$$
\left|F\left(z_{0}\right)\right| \leqq K_{\sigma} N_{\sigma}^{q}(F),
$$

$z_{0}$ in $T$

where the constant $K_{\sigma}$ depends on $q, \sigma$, and $T$, but not on $z_{0}$ or $F(z)$.

Lemma 1.1 is a consequence of the principle of maximum modulus if $q=\infty$ and otherwise is not difficult to prove [5, p. 96].

To proceed with the proof of the theorem for $q<\infty$, let $\epsilon(>0)$ be given. By (1.3) for $q=\infty, t=\infty$, we have for $n$ sufficiently large

$$
\text { [lub } \left.\left|f_{n}(z)\right|, z \text { in } R_{1}\right] \leqq e^{[\gamma(\rho-1)+e] n} \text {, }
$$


whence for the extremal function $F_{n}(z)$

$$
N_{1}^{q}\left(F_{n}\right) \leqq N_{1}^{q}\left(f_{n}\right) \leqq e^{[\gamma(p-1)+\epsilon] n} N_{1}^{q}(1),
$$

and by the Hölder inequality

$$
N_{1}^{t}\left(F_{n}\right) \leqq N_{1}^{q}\left(F_{n}\right) N_{1}^{t}(1) / N_{1}^{q}(1) \leqq e^{[\gamma(\rho-1)+\epsilon] n} N_{1}^{t}(1), \quad 0<t<q .
$$

Thus the first member of (1.3) is not greater than the second member of (1.3) for $\sigma=1$.

To continue with the proof of Theorem 1.1 we introduce points $\alpha_{n 1}, \alpha_{n 2}, \cdots, \alpha_{n n}, n=1,2, \cdots$, uniformly distributed on $C_{1}$ with respect to the conjugate function of $V(z)$ as parameter. It is then known [5, pp. 167$168 ; 7$, pp. $47-48 ; 9$, pp. $271-272$ ] that we have

$$
\lim _{n \rightarrow \infty}\left|\left(z-\alpha_{n 1}\right)\left(z-\alpha_{n 2}\right) \cdots\left(z-\alpha_{n n}\right)\right|^{1 / n}=e^{V_{2}(z)},
$$

uniformly on any closed subset of $R_{1}$, from which there follows by (1.1)

$$
\begin{gathered}
\lim _{n \rightarrow \infty}\left|r_{n}(z)\right|^{1 / n}=e^{\nabla(z)}, \\
r_{n}(z) \equiv \frac{\left(z-\beta_{n 1}\right) \cdots\left(z-\beta_{n n}\right)}{\left(z-\alpha_{n 1}\right) \cdots\left(z-\alpha_{n n}\right)},
\end{gathered}
$$

uniformly on any closed subset of $R_{1}-\bar{R}_{0}$. A consequence of (1.5) for $0<\sigma<\sigma_{1}<1$ and for arbitrary $\epsilon(>0)$ is for $n$ sufficiently large

$$
\begin{aligned}
& \left|r_{n}(z)\right| \leqq e^{[\gamma(1-\sigma)+\epsilon] n}, \\
& z \text { on } C_{\sigma} \text {, } \\
& \left|r_{n}(z)\right| \geqq e^{\left[\gamma\left(1-\sigma_{1}\right)-\epsilon\right] n}, \\
& z \text { on } C_{\sigma_{1}} \text {. }
\end{aligned}
$$

By use of the triangle inequality, by (1.4) and Lemma 1.1 we have

$$
\left|F_{n}(z)-f_{n}(z)\right| \leqq K_{1} e^{[\gamma(\rho-1)+\epsilon] n}, \quad z \text { on } C_{\sigma_{1}} .
$$

But the function $\left[F_{n}(z)-f_{n}(z)\right] / r_{n}(z)$ has only removable singularities in the points $\beta_{n k}$, so by the principle of maximum modulus for the region $R_{\sigma_{1}}$ and by (1.6) we have

$$
\left|F_{n}(z)-f_{n}(z)\right| \leqq K_{2} e^{\left[\gamma\left(\rho-\sigma+\sigma_{1}-1\right)+3 \epsilon\right] n}, \quad z \text { in } R_{\sigma} .
$$

Choose $0<\rho \leqq \sigma<1$. By (1.3) for the case $q=t=\infty$ already established we have

$$
\left|f_{n}(z)\right| \leqq K_{3} e^{[\gamma(\rho-\sigma)+\epsilon] n}, \quad z \text { in } R_{\sigma},
$$

whence by (1.7)

$$
\left|F_{n}(z)\right| \leqq K_{4} e^{\left[\gamma\left(\rho-\sigma+\sigma_{1}-1\right)+3 \epsilon\right] n}, \quad z \text { in } R_{\sigma},
$$

so by the arbitrariness of $\sigma_{1}(<1)$ and $\epsilon(>0)$ the first member of $(1.3)$ is 
not greater than the second member of (1.3).

We turn now to the case $0<\sigma<\rho$. If $\epsilon(>0)$ is arbitrary we have by (1.2) as already established for $q=t=\infty$

$$
\left|f(z)-f_{n}(z)\right| \leqq K_{b} e^{[\gamma(\rho-\sigma)+e] n}, \quad z \text { in } R_{\sigma},
$$

whence from (1.7)

$$
\left|f(z)-F_{n}(z)\right| \leqq K_{6} e^{\left[\gamma\left(p-\sigma+\sigma_{1}-1\right)+3 e\right] n}, \quad z \text { in } R_{\sigma} ;
$$

thus the first member of (1.2) is not greater than the second member. For the case $\sigma=0$ we obtain this same conclusion from the case $\sigma>0$ by integrating the $t$ th power of both members of (1.8) over $R_{0}$, taking the superior limit of the $t n$th root of the first member, and then allowing $\sigma$ to approach zero. That $F_{n}(z)$ converges to $f(z)$ throughout $R_{p}$, uniformly on any closed subset of $R_{p}$, follows from (1.2) with $t=\infty$.

The fact that the first member of (1.2) is not less than the second member of (1.2) for $\sigma>0$ and that the first member of (1.3) is not less than the second member of (1.3) can now be proved by standard methods [7, p. 50], assuming the contrary, using Lemma 1.1 to study the sequence of functions involved on an auxiliary level locus $C_{\sigma_{1}}$ near $C_{\sigma}, \sigma_{1}<\sigma$, and applying the two-constant theorem in $R_{\sigma_{1}}-\bar{R}_{0}$ to show that $f(z)$ is analytic throughout some $R_{\rho^{\prime}}, \rho^{\prime}>\rho$. The case $\sigma=0$ in (1.2) is exceptional here, but can be treated similarly by using an auxiliary set of Jordan curves $C_{0}^{\prime}$ interior to $R_{0}$ instead of the locus $C_{\sigma_{1}}$; when $C_{0}^{\prime}$ approaches $C_{0}$, the analog $\phi^{\prime}(z)$ of $\phi(z)$ approaches [4] the function $\phi(z)$ uniformly throughout $\bar{R}_{1}-R_{0}$, and the reasoning already outlined applies in essence.

Theorem 1.1, whose proof is now complete, can be generalized by inserting positive continuous weight functions in the integrals of (1.2) and (1.3). Several corollaries of Theorem 1.1 can be readily proved by the reader:

COROLlaRy 1.1. For any sequence of functions $\left\{F_{n}^{*}(z)\right\}$ of the respective classes $\mathcal{L}_{n}^{q}\left(R_{1}\right)$ we have for $q \leqq t \leqq \infty$

$$
\limsup _{n \rightarrow \infty}\left[N_{\sigma}^{t}\left(F_{n}^{*}\right)\right]^{1 / n} \geqq e^{\gamma(\rho-\sigma)}, \quad \rho<\sigma \leqq 1 .
$$

Corollary 1.2. Equality in (1.2) and (1.3) holds for every subsequence $\left\{F_{n_{k}}(z)\right\}$ with lim $\sup _{k \rightarrow \infty}\left(n_{k+1}-n_{k}\right)<\infty$.

COROLlaRy 1.3. For any sequence of functions $\left\{F_{n_{k}}^{*}(z)\right\}$ of the respective classes $\mathcal{L}_{n_{k}}^{a}\left(R_{1}\right)$ with lim $\sup _{k \rightarrow \infty}\left(n_{k+1}-n_{k}\right)<\infty$ we have for $q \leqq t \leqq \infty$

$$
\limsup _{n_{k} \rightarrow \infty}\left[N_{\sigma}^{t}\left(F_{n_{k}}^{*}\right)\right]^{1 / n_{k}} \geqq e^{\gamma(\rho-\sigma)}, \quad \rho<\sigma \leqq 1 .
$$

Corollary 1.4. Let the function $f(z)(\not \equiv 0)$ of Theorem 1.1 be analytic throughout $R_{1}$. Then the extremal functions $F_{n}(z)$ converge to $f(z)$ throughout $R_{1}$, 
uniformly on any closed subset of $R_{1}$, and we have for all $t(>0)$

and for $0<t \leqq q$

$$
\limsup _{n \rightarrow \infty}\left[N_{\sigma}^{t}\left(f-F_{n}\right)\right]^{1 / n} \leqq e^{\gamma(1-\sigma)}, \quad 0 \leqq \sigma<1,
$$

$$
\limsup _{n \rightarrow \infty}\left[N_{1}^{t}\left(F_{n}\right)\right]^{1 / n}=1
$$

2. Interpolation by functions of minimum norm, parametric integrals. We now modify the problem considered in $\S 1$ by replacing the surface integrals by integrals over the loci $C_{\sigma}, 0 \leqq \sigma \leqq 1$, taken with respect to $\psi(z)$ as parameter, where $\psi(z)$ is the conjugate of $\phi(z)$ in $R_{1}-\bar{R}_{0}$.

We employ the geometric situation and notation of Theorem 1.1. Let $q$ be given, $0<q \leqq \infty$. The function $G(z)$ is said to belong to the class $\mathcal{H}^{q}$ if $G(z)$ is analytic in $R_{1}$ and if the norms

$$
N_{\sigma}^{q}(G) \equiv\left[\int_{C_{\sigma}}|G(z)|^{q} d \psi(z)\right]^{1 / q}, \quad 0 \leqq \sigma<1,
$$

are bounded, where $N_{\sigma}^{\infty}(G)$ is interpreted as $\left[\max |G(z)|, z\right.$ on $\left.C_{\sigma}\right]$. The functions $G(z)$ of class $\mathcal{X}^{a}$ have two important properties [11]:

A. For all points $\zeta$ of $C_{1}$, with the possible exception of a set of measure zero (with respect to $\psi$ ), $\lim G(z)$ exists when $z$ in $R_{1}$ approaches $\zeta$ along a level curve of $\psi(z)$. Moreover for these limit values $\int_{C_{1}}|G(\zeta)|^{a} d \psi$ exists in the sense of Lebesgue, and $(0<q<\infty)$

$$
\lim _{\sigma \rightarrow 1} N_{\sigma}^{q}(G)=N_{1}^{q}(G),
$$

where $N_{1}^{q}(G)$ is defined by the analog of (2.1). With $q=\infty$ analogous (Fatou) properties are well known.

B. The function $\log N_{\sigma}^{q}(G)$ is a convex function of $\sigma, 0 \leqq \sigma \leqq 1$, in the sense

$$
N_{\sigma}^{q}(G) \leqq\left[N_{1}^{q}(G)\right]^{\sigma}\left[N_{0}^{q}(G)\right]^{1-\sigma} .
$$

Let $\mathcal{H}_{n}^{q}$ denote that subclass of $\mathcal{H}^{q}$ consisting of those functions which coincide with the given function $f(z)$ in the points $\beta_{n 1}, \cdots, \beta_{n n}$. We prove later the existence of a function $G_{n}(z)$ of class $\mathcal{X}_{n}^{q}$ of minimum norm.

Theorem 2.1. Under the hypothesis of Theorem 1.1 on $C_{0}, C_{1}, f(z), \rho$, and the points $\beta_{n k}$, for given $q(0<q \leqq \infty)$ the extremal functions $G_{n}(z)$ converge to $f(z)$ throughout $R_{\rho}$, uniformly on any closed subset of $R_{\rho}$, and we have for all $t(>0)$ with the notation (2.1)

$$
\limsup _{n \rightarrow \infty}\left[N_{\sigma}^{t}\left(f-G_{n}\right)\right]^{1 / n}=e^{\gamma(\rho-\sigma)}, \quad 0 \leqq \sigma<\rho,
$$

and for all $t(>0)$ if $\rho \leqq \sigma<1$ and for $0<t \leqq q$ if $\sigma=1$, 


$$
\limsup _{n \rightarrow \infty}\left[N_{0}^{t}\left(G_{n}\right)\right]^{1 / n}=e^{\gamma(p-\sigma)} .
$$

We shall need the following analog of Lemma 1.1:

Lemma 2.1. Let $C_{0}$ and $C_{1}$ satisfy the hypothesis of Theorem 1.1, let $G(z)$ be an arbitrary function of class $\mathcal{X}\left(a\right.$, and let $T$ be an arbitrary closed subset of $R_{\sigma}$, $0<\sigma \leqq 1$, where no critical point of $\phi(z)$ lies on $C_{\sigma}(0<\sigma<1)$. Then we have

$$
\left|G\left(z_{0}\right)\right| \leqq K_{\sigma} N_{\sigma}^{q}(G),
$$

$z_{0}$ in $T$,

where the constant $K_{\sigma}$ depends on $q, \sigma$, and $T$, but not on $z_{0}$ or $G(z)$.

If $q=\infty$, the lemma follows from the maximum principle, so henceforth in the proof we assume $0<q<\infty$; we assume also without loss of generality $\sigma=1$. Under a smooth conformal map of $R_{1}$ onto a region bounded by a finite number of disjoint analytic Jordan curves the functions $\phi(z)$ and $\psi(z)$ are invariant, and hence $N_{\sigma}^{q}$ is also invariant. Thus we may and do assume in the proof of Lemma 2.1 that $C_{1}$ consists of a finite number of mutually disjoint analytic Jordan curves; it follows that $\phi(z)$ and $\psi(z)$ are harmonic on $C_{1}$, and $\phi(z)$ has no critical points on $C_{1}$. Indeed, we suppose that $\phi(z)$ has no critical points also on the set $R_{1}-\bar{R}_{1-\eta}$, where $\eta(>0)$ is suitably chosen, and suppose $T$ interior to $R_{1-\eta}$.

For $1-\eta<\sigma<1$ the function $|G(z)|^{q}$ is subharmonic in $R_{\sigma}$, and we can write

$$
\left|G\left(z_{0}\right)\right|^{q} \leqq \frac{1}{2 \pi} \int_{C_{\sigma}}|G(z)|^{q} \frac{\partial g}{\partial \nu} d s, \quad z_{0} \text { in } T,
$$

where $g\left(z, z_{0}\right)$ is Green's function for the region $R_{\sigma}$ with pole in $z_{0}$, and $\nu$ is the inner normal for $R_{\sigma}$. The normal derivatives $\partial g / \partial \nu$ are uniformly bounded for $z_{0}$ in $T$ and for $1-\eta<\sigma<1$, as the reader may show. Moreover, the directional derivatives $\partial \phi / \partial \nu=-\partial \psi / \partial s$ are uniformly bounded from zero on $C_{\sigma}, 1-\eta<\sigma<1$. From (2.5) we can write

$$
\left|G\left(z_{0}\right)\right| \leqq K_{1} N_{\sigma}^{q}(G) \text {, }
$$

where $K_{1}$ is independent of $\sigma, 1-\eta<\sigma<1$. Approach of $\sigma$ to unity now yields (2.4) for $\sigma=1$, so Lemma 2.1 is established.

We are now in a position to establish the existence of an extremal function of class $\mathscr{X}_{n}^{q}$. It follows from Lemma 2.1 that a set of functions of class $\mathscr{X}_{n}^{q}$ all of norm less than $M(<\infty)$ is normal in $R_{1}$, and the equation $G^{k}(z) \rightarrow G^{0}(z)$ uniformly on any closed set interior to $R_{1}$ implies

$$
N_{\sigma}^{q}\left(G^{0}\right) \leqq \lim \inf N_{\sigma}^{q}\left(G^{k}\right), \quad 0 \leqq \sigma<1,
$$

where the $G^{k}(z)$ are in $\mathscr{H}_{n}^{\ell}$. We choose the $G^{k}(z)$ with 


$$
N_{1}^{q}\left(G^{k}\right) \rightarrow \inf \left[N_{1}^{q}(G), G \text { in } \mathcal{X}_{n}^{q}\right]=M_{n},
$$

and with $G^{k}(z) \rightarrow G^{0}(z)$ uniformly on any closed set interior to $R_{1}$. For arbitrary $\epsilon(>0)$ and for $k$ sufficiently large we have $N_{1}^{q}\left(G^{k}\right)<M_{n}+\epsilon$, whence by Lemma 2.1 with $\sigma=1$ and $T=C_{0}$

$$
N_{0}^{q}\left(G^{k}\right) \leqq K_{2}\left(M_{n}+\epsilon\right) .
$$

Thus by property $\mathrm{B}$

$$
N_{\sigma}^{q}\left(G^{k}\right) \leqq\left(M_{n}+\epsilon\right)^{\sigma}\left[K_{2}\left(M_{n}+\epsilon\right)\right]^{1-\sigma}
$$

so for $\sigma$ sufficiently near unity we have

$$
N_{\sigma}^{q}\left(G^{k}\right) \leqq M_{n}+2 \epsilon .
$$

The corresponding inequality holds for $G^{0}$ :

$$
N_{\sigma}^{q}\left(G^{0}\right) \leqq M_{n}+2 \epsilon .
$$

Hence $G^{0}$ is of class $\mathscr{H}_{n}^{Q}$, and we have

$$
N_{1}^{q}\left(G^{0}\right) \leqq M_{n},
$$

but the strong inequality is impossible.

The extremal function $G_{n}(z)$ of class $\mathcal{X}_{n}^{q}$ is unique if $1<q<\infty$, for if two functions of class $\mathcal{X}_{n}^{q}$ have the same norm, half their sum has a smaller norm.

The proof of Theorem 2.1 now follows directly the proof of Theorem 1.1 by using Lemma 2.1 (which applies for all $\sigma(>0)$ with at most a finite number of exceptions) instead of Lemma 1.1. The impossibility of inequality instead of equality in (2.2) and (2.3) is proved using property B instead of the two-constant theorem.

3. Interpolation by functions of minimum norm, line integrals. We now modify the problem considered in $\$ 1$ by replacing the surface integrals by line integrals over the loci $C_{\sigma}, 0 \leqq \sigma \leqq 1$, with respect to arc length. With the hypothesis of Theorem 1.1, let the components of $C_{1}$ be rectifiable Jordan curves. For given $q, 1<q<\infty$, the function $H(z)$ is said to belong to the class $\mathcal{E}^{q}$ if $H(z)$ is analytic in $R_{1}$ and can be represented there by an integral

$$
H\left(z_{0}\right) \equiv \frac{1}{2 \pi i} \int_{C_{1}} \frac{H_{1}(z) d z}{z-z_{0}},
$$

$z_{0}$ in $R_{1}$,

where $H_{1}(z)$ is of class $\mathcal{L}^{q}$, namely $H_{1}(z)$ is measurable and $\left|H_{1}(z)\right|^{q}$ is Lebesgue integrable with respect to arc length on $C_{1}$. The function $H_{1}(z)$ in (3.1) is not uniquely determined. The class $\mathcal{E}^{a}$ is similar to a class which was first introduced by Garabedian [3].

For each $n$ let $\mathcal{L}_{n}^{q}$ denote the subclass of $\mathcal{L}^{q}$ consisting of all functions 
$H_{n 1}(z)$ corresponding to functions $H_{n}(z)$ in the representation (3.1) which coincide with the given $f(z)$ in the points $\beta_{n 1}, \beta_{n 2}, \cdots, \beta_{n n}$. The class $\mathcal{L}_{n}^{q}$ contains all polynomials which interpolate to $f(z)$ in these points, hence is nonempty. There exists a function $H_{n 1}^{*}(z)$ of class $\mathcal{L}_{n}^{q}$, essentially unique on $C_{1}$, such that the norm

$$
N_{1}^{q}\left(H_{n 1}^{*}\right)=\left[\int_{C_{1}}\left|H_{n 1}^{*}(z)\right|^{q}|d z|\right]^{1 / q}
$$

is a minimum among all functions of class $\mathcal{L}_{n}^{q}$. This existence follows [3] by the completeness and uniform convexity [Clarkson, 2] of the class $\mathcal{L}^{q}$, and by the closure and convexity of the subclass $\mathcal{L}_{n}^{a}$. The uniqueness of $H_{n 1}^{*}(z)$ follows easily; compare $\$ 2$. We denote by $H_{n}^{*}(z)$ the function of class $\varepsilon^{a}$ which corresponds to $H_{n 1}^{*}(z)$ by the analog of (3.1). Our main result, with notation $N_{\sigma}^{q}$ analogous to (3.2), is

Theorem 3.1. Let $C_{0}, C_{1}, \beta_{n k}, f(z)$, and $\rho$ satisfy the conditions of Theorem 1.1 , and suppose in addition that $C_{0}$ and $C_{1}$ are rectifiable. Then for fixed $q$, $1<q<\infty$, the functions $H_{n}^{*}(z)$ of class $\varepsilon_{q}$ converge to $f(z)$ throughout $R_{p}$, uniformly on any closed subset of $R_{\rho}$, and we have for all $t(>0)$

and for $0<t \leqq q$

$$
\begin{array}{rlrl}
\underset{n \rightarrow \infty}{\limsup }\left[N_{\sigma}^{t}\left(f-H_{n}^{*}\right)\right]^{1 / n} & =e^{\gamma(\rho-\sigma)}, & & 0 \leqq \sigma<\rho, \\
\limsup _{n \rightarrow \infty}\left[N_{\sigma}^{t}\left(H_{n}^{*}\right)\right]^{1 / n}=e^{\gamma(\rho-\sigma)}, & \rho \leqq \sigma<1,
\end{array}
$$

$$
\limsup _{n \rightarrow \infty}\left[N_{1}^{t}\left(H_{n 1}^{*}\right)\right]^{1 / n}=e^{\gamma(p-1)} .
$$

The lemmas of $\S \S 1$ and 2 have an analog here:

Lemma 3.1. Under the conditions of Theorem 3.1 on $C_{0}$ and $C_{1}$, let $H(z)$ be an arbitrary function of class $\mathcal{E}^{q}$ and $H_{1}(z)$ a function of class $\mathcal{L}^{a}$ corresponding to $H(z)$ in (3.1). If $T$ is any closed set interior to $R_{\sigma}$, we have

$$
\begin{aligned}
& \left|H\left(z_{0}\right)\right| \leqq K_{\sigma} N_{\sigma}^{q}(H), \\
& \left|H\left(z_{0}\right)\right| \leqq K_{1} N_{1}^{q}\left(H_{1}\right), \quad z_{0} \text { in } T, \sigma=1 \text {, }
\end{aligned}
$$

where the constant $K_{\sigma}$ depends on $q, \sigma$, and $T$, but not on $z_{0}, H(z)$, or $H_{1}(z)$.

Lemma 3.1 follows by the Hölder inequality from Cauchy's integral formula for $0 \leqq \sigma<1$ and from (3.1) for $\sigma=1$.

If the components of $C_{1}$ in Theorem 3.1 are analytic Jordan curves, the function $f_{n}(z)$ (notation of $\S 1$ ) is of class $\mathcal{E} q$, for limit values of $f_{n}(z)$ bounded in their totality exist almost everywhere on $C_{1}$, and $f_{n}(z)$ is represented in $R_{1}$ by the corresponding Cauchy integral. The functions $f_{n}(z)$ can then be 
used as comparison functions, and the proof of Theorem 1.1 essentially applies in the present situation.

To prove Theorem 3.1 in the case that the components of $C_{1}$ are arbitrary rectifiable Jordan curves, we replace the points $\alpha_{n k}$ of $\$ 1$ by suitably chosen new points $\alpha_{n \mathfrak{z}}^{\prime}$ on a set of analytic Jordan curves $C_{1}^{\prime}$ exterior to $R_{1}$ but geometrically near $C_{1}$. The comparison functions $f_{n}(z)$ of $\$ 1$ are replaced by rational functions $R_{n}(z)$ interpolating to $f(z)$ in the $\beta_{n k}$ and with poles in the $\alpha_{n k}^{\prime}$. The curves $C_{1}^{\prime}$ can be so chosen [7, pp. 48-49] that the asymptotic properties of the $\alpha_{n k}^{\prime}$ and of the $R_{n}(z)$ differ as little as we please from the asymptotic properties of the $\alpha_{n k}$ and of the $f_{n}(z)$ respectively. With these modifications, the method of proof of Theorem 1.1 is essentially valid to establish Theorem 3.1.

4. Series of interpolation. In the respective situations of Theorems 1.1, 2.1 , and 3.1, with $q=2$ and $\beta_{n k}$ independent of $n$, the general theory of orthogonal functions can be used; each theorem mentioned leads to a unique formal expansion of $f(z)$ which can be defined by interpolation to $f(z)$ in the points $\beta_{k}$. For definiteness we restrict ourselves in our detailed discussion to the situation and method of $\S 1$.

We assume for the present that each $\beta_{k}$ lies in $R_{1}$, but do not assume (1.1). Let $\phi_{n}(z)$ be the function of class $\mathcal{L}^{2}\left(R_{1}\right)$ satisfying the conditions of interpolation

$$
\phi_{n}\left(\beta_{1}\right)=\phi_{n}\left(\beta_{2}\right)=\cdots=\phi_{n}\left(\beta_{n-1}\right)=0, \quad \phi_{n}\left(\beta_{n}\right)=1,
$$

and which minimizes $N_{1}^{2}\left(\phi_{n}\right)$ over the class $\mathcal{L}^{2}\left(R_{1}\right)$. If the points $\beta_{1}, \beta_{2}, \cdots, \beta_{n}$ are not all distinct, equations (4.1) and later statements require special interpretation, as is customary in the theory of interpolation. Let $\phi_{n}^{*}(z)$ be defined as $\phi_{n}(z) / N_{1}^{2}\left(\phi_{n}\right)$. In the important case that all $\beta_{k}$ are identical, these functions were introduced by Bergman [1]; in the case that the sequence $\left\{\beta_{n}\right\}$ approaches a limit, these functions were studied by Walsh and Davis [10]; existence and uniqueness of the $\phi_{n}^{*}(z)$ follow from $\$ 1$; the fact that the $\phi_{n}^{*}(z)$ are mutually orthogonal over $R_{1}$ follows readily $[1 ; 10$; compare 12$]$, as does the fact that $\phi_{n}^{*}(z)$ is orthogonal to any function of class $\mathcal{L}^{2}\left(R_{1}\right)$ which vanishes in the points $\beta_{1}, \beta_{2}, \cdots, \beta_{n}$. Of course $\phi_{n}^{*}(z)$ also is an extremal function, namely the function of class $\mathcal{L}^{2}\left(R_{1}\right)$ of norm unity which vanishes in $\beta_{1}, \beta_{2}, \cdots, \beta_{n-1}$ whose value in $\beta_{n}$ is positive and maximum.

An arbitrary function $F(z)$ of class $\mathcal{L}^{2}\left(R_{1}\right)$ possesses two formal expansions in terms of the $\phi_{n}^{*}(z)$; the one is

$$
F(z) \sim a_{1} \phi_{1}^{*}(z)+a_{2} \phi_{2}^{*}(z)+\cdots, a_{n}=\iint_{R_{1}} F(z) \bar{\phi}_{n}^{*}(z) d S,
$$

where the coefficients $a_{n}$ are found by the usual orthogonal function (Fourier) method; the other is a series of interpolation 


$$
F(z) \sim b_{1} \phi_{1}^{*}(z)+b_{2} \phi_{2}^{*}(z)+\cdots,
$$

where $b_{1}$ is determined by setting formally $z=\beta_{1}$ in (4.3), then $b_{2}$ is determined by setting formally $z=\beta_{2}$ in (4.3), etc. These two formal expansions are identical, independently of the completeness of the set of functions $\phi_{n}^{*}(z)$; for the proof compare the corresponding discussion for harmonic functions [12]. If the functions $\phi_{n}^{*}(z)$ form a complete set, for which it is sufficient that the points $\beta_{k}$ have at least one limit point interior to $R_{1}$, the formal expansion (4.2) converges to $F(z)$ throughout $R_{1}$, uniformly on any closed subset of $R_{1}$; if the functions $\phi_{n}^{*}(z)$ do not form a complete set, the formal expansion (4.2) converges throughout $R_{1}$, uniformly on any closed subset of $R_{1}$, to the function of minimum norm which coincides with $F(z)$ in all the points $\beta_{k}$. If values $F\left(\beta_{k}\right)$ are given without the hypothesis of existence of a function $F(z)$ of class $\mathcal{L}^{2}\left(R_{1}\right)$ which takes those values in the respective points $\beta_{k}$, the formal expansion (4.3) still exists, and a necessary and sufficient condition for the existence of such a function $F(z)$ is the convergence of the series $\sum\left|b_{n}\right|^{2}$, where the $b_{n}$ are defined as in (4.3); if this condition is satisfied, the series (4.3) converges in the mean (of order two) in $R_{1}$, thus converges throughout $R_{1}$, uniformly on any closed subset of $R_{1}$, and to the function of class $\mathcal{L}^{2}\left(R_{1}\right)$ of least norm which takes on the prescribed values in the points $\beta_{k}$. In particular the sum of the first $n$ terms of the series in (4.3) is the function of $\mathcal{L}^{2}\left(R_{1}\right)$ of least norm which takes on the prescribed values in the points $\beta_{1}, \beta_{2}, \cdots, \beta_{n}$.

We now return to the hypothesis (1.1), and shall prove the validity of an interpolation series expansion under suitable conditions for functions not necessarily of class $\mathcal{L}^{2}\left(R_{1}\right)$.

Theorem 4.1. Let $R_{0}, R_{1}, C_{0}, C_{1}$, and the points $\beta_{k}$ (independent of $n$ ) satisfy the conditions of Theorem 1.1. If $f(z)$ is analytic throughout $R_{\sigma}$, then the expansion

$$
f(z)=\sum_{n=1}^{\infty} a_{n} \phi_{n}^{*}(z),
$$

where the $a_{n}$ are determined formally by interpolation in the points $\beta_{k}$, is valid in $R_{\sigma}$, uniformly on any closed subset of $R_{\sigma}$.

If $f^{\prime}(z)$ is analytic throughout $R_{\rho}, 0<\rho<1$, but not throughout any $R_{\rho^{\prime}}, \rho^{\prime}>\rho$, then we have

$$
\limsup _{n \rightarrow \infty}\left|a_{n}\right|^{1 / n}=e^{\gamma(\rho-1)},
$$

and if $f(z)$ is analytic throughout $R_{1}$, the first member of (4.5) is not greater than unity. Conversely, if the second member of (4.4) is given with (4.5) valid, $0<\rho<1$, the series converges throughout $R_{\rho}$, uniformly on any closed subset of $R_{p}$, to a function $f(z)$ analytic throughout $R_{p}$ but not analytic throughout any $R_{\boldsymbol{\rho}^{\prime}}, \boldsymbol{\rho}^{\prime}>\rho$; this function $f(z)$ has (4.4) as its formal expansion found by inter- 
polation in the points $\beta_{n}$. Likewise if the $a_{n}$ are given with the first member of (4.5) not greater than unity, the series in (4.4) converges throughout $R_{1}$, uniformly on any closed subset of $R_{1}$, to a function $f(z)$ analytic throughout $R_{1}$; this function $f(z)$ has (4.4) as its formal expansion found by interpolation in the points $\beta_{n}$.

If functional values $f\left(\beta_{n}\right)$ are given without assuming the existence of $f(z)$ other than in the points $\beta_{n}$, a formal development (4.4) exists; equation (4.5), with $0<\rho<1$, is a necessary and sufficient condition for the existence of a function $f(z)$ taking on the prescribed values in the points $\beta_{n}$, analytic interior to $R_{\rho}$ but not analytic interior to any $R_{\rho^{\prime}}, \rho^{\prime}>\rho$; likewise that the first member of (4.5) be not greater than unity is a necessary and sufficient condition for the existence of a function $f(z)$ taking on the prescribed values in the points $\beta_{n}$, analytic throughout $R_{1}$.

We have already remarked that (notation of $\$ 1$ )

$$
F_{n}(z) \equiv \sum_{k=1}^{n} a_{k} \phi_{k}^{*}(z)
$$

so the validity of (4.4) follows from Theorem 1.1.

To study the numbers $a_{n}$, we write

$$
a_{n}=\iint_{R_{1}} F_{n}(z) \bar{\phi}_{n}^{*}(z) d S,\left|a_{n}\right| \leqq N_{1}^{2}\left(F_{n}\right),
$$

by Schwarz's inequality. It follows from (1.3) and Corollary 1.4 that the first member of (4.5) is not greater than the second member, even if $\rho=1$.

To prove equality in (4.5) we need to consider the asymptotic behavior of the $\phi_{n}^{*}(z)$. By Lemma 1.1 the functions $\left|\phi_{n}^{*}(z)\right|$ are uniformly bounded by some $K_{1}$ on any closed subset $T$ of $R_{1}$. If $r_{n}(z)$ has the meaning of $\S 1$, for given $\epsilon(>0)$ and for $0<\sigma<\sigma_{1}<1$, we have for $n$ sufficiently large

$$
\begin{array}{lll}
\left|r_{n-1}(z)\right| \geqq e^{\left[\gamma\left(1-\sigma_{1}\right)-\epsilon\right](n-1)}, & & z \text { on } C_{\sigma_{1}}, \\
\left|r_{n-1}(z)\right| \leqq e^{[\gamma(1-\sigma)+\epsilon](n-1)}, & & z \text { on } C_{\sigma} .
\end{array}
$$

The function $\phi_{n}^{*}(z) / r_{n-1}(z)$ is analytic in $R_{1}$ when suitably defined in the points $\beta_{k}$, so by the maximum principle

$$
\left|\phi_{n}^{*}(z)\right| \leqq K_{1} e^{\left[\gamma\left(\sigma_{1}-\sigma\right)+2 \epsilon\right](n-1)}, \quad \quad z \text { in } \bar{R}_{\sigma} .
$$

Thus we may write $\left(\epsilon \rightarrow 0, \sigma_{1} \rightarrow 1\right)$

$$
\limsup _{n \rightarrow \infty}\left[\max \left|\phi_{n}^{*}(z)\right|, z \text { on } \bar{R}_{\sigma}\right]^{1 / n} \leqq e^{\gamma(1-\sigma)}, \quad 0<\sigma<1 .
$$

Since $f(z)$ is analytic throughout no $R_{\rho^{\prime}}, \rho^{\prime}>\rho$, the series in (4.4) can converge uniformly throughout no $R_{\rho^{\prime}}$, so we deduce from (4.6) and from the part of (4.5) already proved the validity of (4.5).

The remainder of Theorem 4.1 follows without difficulty by continued 
use of (4.6). Theorem 4.1 was previously established [10] by a somewhat different method for the case that $\beta_{k}$ approaches a point of $R_{1}$.

Theorem 4.1 considers series of interpolation which are series of orthogonal functions according to the orthogonality of $\$ 1$; the corresponding discussions for orthogonality as measured in $\$ \S 2$ and 3 presents no difficulty; proofs of the precise analogs of Theorem 4.1 are left to the reader.

5. Asymptotic behavior of orthonormal functions and of their zeros. Methods previously used by the present authors [13] in the study of zeros of extremal polynomials apply also in the study of the extremal functions $\phi_{n}^{*}(z)$ and their analogs.

If the function $U(z)$ is harmonic in a region $R$, and if each of the functions $h_{n}(z)$ is locally single-valued and analytic in $R$ except perhaps for branch points, with $\left|h_{n}(z)\right|$ single-valued in $R$, we say that $U(z)$ is a harmonic majorant of the sequence $\left\{h_{n}(z)\right\}$ in $R$ if for every continuum $Q$ (not a single point) in $R$ we have

$$
\limsup _{n \rightarrow \infty}\left[\max \left|h_{n}(z)\right|, z \text { on } Q\right] \leqq\left[\max e^{U(z)}, z \text { on } Q\right] ;
$$

and $U(z)$ is an exact harmonic majorant of the sequence $\left\{h_{n}(z)\right\}$ in $R$ if (5.1) is replaced by equality of the two members, for every continuum $Q$ (not a single point) in $R$.

Again we devote our attention to the situation of $\S 1$, but the methods and results appy equally to the situations of $\S \S 2$ and 3 .

THEOREM 5.1. Under the conditions of Theorem 4.1 the function $U(z)$ $\equiv \gamma[1-\phi(z)]$ is an exact harmonic majorant of the sequence $\left[\phi_{n}^{*}(z)\right]^{1 / n}$ and of every subsequence in $R_{1}-\bar{R}_{0}$.

Equality holds in (4.6) for every $\sigma, 0<\sigma<1$; for suppose the strong inequality to hold for some $\sigma$, say

$$
\left|\phi_{n}^{*}(z)\right| \leqq K_{1} e^{\gamma n\left(1-\sigma_{1}\right)}, \quad z \text { on } C_{\sigma}, 0<\sigma_{1}<\sigma ;
$$

then we choose $\rho, \sigma_{1}<\rho<\sigma$, and set $a_{n}=e^{\gamma n(\rho-1)}$. The series $\sum a_{n} \phi_{n}^{*}(z)$ converges to a function analytic throughout $\bar{R}_{\sigma}$, in contradiction to Theorem 4.1. It now follows [8, Corollary 2 to Theorem 4 ] that $U(z)$ is an exact harmonic majorant of the sequence $\left[\phi_{n}^{*}(z)\right]^{1 / n}$ in $R_{1}-\bar{R}_{0}$.

If $U(z)$ is not an exact harmonic majorant for every subsequence of the sequence $h_{n}(z)$, the strong inequality must hold [8, Corollary to Theorem 1] in (5.1) for some subsequence of the $h_{n}(z)$, for every $Q$ in $R$. If $\gamma[1-\phi(z)]$ is not an exact harmonic majorant in $R_{1}-\bar{R}_{0}$ for every subsequence of the $\left[\phi_{n}^{*}(z)\right]^{1 / n}$, inequality (5.2) holds for some subsequence $\phi_{n_{k}}^{*}(z)$, and we reach a contradiction as before by setting $a_{n_{k}}=e^{\gamma n_{k}(\rho-1)}, a_{n}=0$ if $n \neq n_{k}$. Theorem 5.1 is established.

We now state two immediate results [8, Theorem 16 and Remark 1; compare also 13]. 
THEOREM 5.2. Under the conditions of Theorem 4.1 let $R$ be any subregion of $R_{1}-\bar{R}_{0}$, and let $N_{n}$ indicate the number of zeros of $\phi_{n}^{*}(z)$ in $R$. Then we have

$$
\lim _{n \rightarrow \infty} N_{n} / n=0 .
$$

THEOREM 5.3. Under the conditions of Theorem 4.1 let $R$ be a subregion of $R_{1}-\bar{R}_{0}$ containing no limit points of the totality of zeros of the $\phi_{n}^{*}(z)$. Then in $R$ we have

$$
\lim _{n \rightarrow \infty}\left|\phi_{n}^{*}(z)\right|^{1 / n}=e^{\gamma[1-\phi(z)]},
$$

uniformly on any closed subregion of $R$.

Theorems 5.1 and 5.3 are of significance in the study of the divergence of the series of Theorem 4.1.

THEOREM 5.4. With the hypothesis of Theorem 4.1, the series in (4.4), where (4.5) is valid with $0<\rho<1$, diverges at an arbitrary point of $R_{1}-\bar{R}_{\rho}$ which is not a limit point of the totality of zeros of the $\phi_{n}^{*}(z)$, and this series converges uniformly on no continuum (not a single point) in $R_{1}-\bar{R}_{\rho}$.

The first part of Theorem 5.4 follows at once from (4.5) and Theorem 5.3; the latter part follows by methods used in detail elsewhere [13, remark subsequent to equation (15) ].

The function $\phi_{n}^{*}(z)$ vanishes by definition in the points $\beta_{1}, \beta_{2}, \cdots, \beta_{n-1}$, but in the case of multiply connected regions $R_{1}$ there may presumably exist other, nontrivial, zeros of $\phi_{n}^{*}(z)$ in $R_{1}$. Theorem 5.2 contains some information on the numbers of these nontrivial zeros. We proceed to indicate a further result, which applies to the norms not of $\S 1$ but to those of $\S \S 2$ and 3; equation (1.1) is not assumed.

Theorem 5.5. With the hypothesis of Theorem 1.1 on $R_{1}$, each $\beta_{n k}$ independent of $n$, and $N_{1}^{2}(G)$ defined by (2.1), let $\chi_{n}(z)$ be the function of class $\mathcal{X}^{2}$ with $\chi_{n}\left(\beta_{1}\right)=\chi_{n}\left(\beta_{2}\right)=\cdots=\chi_{n}\left(\beta_{n-1}\right)=0, \chi_{n}\left(\beta_{n}\right)=1$, whose norm $N_{1}^{2}(\chi)$ is least. $A$ circle whose interior lies in $R_{1}$ and contains $\beta_{n}$ can contain no nontrivial zero of $\chi_{n}(z)$. Consequently any circle whose interior lies in $R_{1}$ and contains all the $\beta_{n}$ contains no nontrivial zero of any $\chi_{n}(z)$. If we have $\beta_{n} \rightarrow \beta_{0}, \beta_{0}$ in $R_{1}$, any circle whose interior lies in $R_{1}$ and contains $\beta_{0}$ can contain no nontrivial zero of $\chi_{n}(z)$ for $n$ sufficiently large.

Let $\alpha$ be a nontrivial zero of $\chi_{n}(z)$ in $R_{1}$. The function $\left(z-\beta_{n}\right) \chi_{n}(z) /(z-\alpha)$ is analytic throughout $R_{1}$ when suitably defined for $z=\alpha$, vanishes in all the points $\beta_{1}, \beta_{2}, \cdots, \beta_{n}$, and is of class $\mathscr{X}^{2}$. Consequently (compare $\S 4$ ) this function is orthogonal to $\chi_{n}(z)$ on $C_{1}$ :

$$
\int_{C_{1}} \bar{\chi}_{n}(z) \frac{\left(z-\beta_{n}\right) \chi_{n}(z)}{z-\alpha} d \psi=0 .
$$


We make the substitution $z^{\prime}=1 /\left(z-\beta_{n}\right), \alpha^{\prime}=1 /\left(\alpha-\beta_{n}\right)$, whence for the image $C_{1}^{\prime}$ of $C_{1}\left(\alpha^{\prime} \neq 0\right)$

$$
\int_{c_{1}^{\prime}}\left|\chi_{n}(z)\right|^{2} \frac{d \psi}{\alpha^{\prime}-z^{\prime}}=0 .
$$

Thus $\alpha^{\prime}$ is a position of equilibrium in the field of force due to a spread of nonnegative matter over $C_{1}^{\prime}$ which repels according to the law of inverse distance, so $\left[9, \S 7.1\right.$, Theorem 1 and $\$ 7.2$, Theorem 1] $\alpha^{\prime}$ lies in the convex hull of $C_{1}^{\prime}$. Consequently if the exterior of any circle contains no point of $C_{1}^{\prime}$, that exterior contains no point $\alpha^{\prime}$. Interpretation of this conclusion in the original $z$-plane yields the theorem.

This theorem is not invariant under arbitrary one-to-one conformal map of $R_{1}$. That is to say, an arbitrary such map of $R_{1}$ which carries $C_{1}$ into a set of mutually disjoint Jordan curves does not necessarily transform a circle in $R_{1}$ into a circle. The conclusion of the theorem applies not merely to a circle, but to any Jordan curve which is the image of a circle under a one-to-one conformal map of $R_{1}$ which transforms $C_{1}$ into a set of mutually disjoint Jordan curves.

Theorem 5.5 extends at once to the situation of $\$ 3$, as does the supplementary remark regarding the image of a circle, now with the condition that the transformation of $R_{1}$ shall transform $C_{1}$ into a set of mutually disjoint rectifiable Jordan curves. Theorem 5.5 is of obvious significance in connection with the analogs of Theorems 5.3 and 5.4.

Theorem 5.5 does not extend without fundamental modification of proof or conclusion to the situation of $\$ 1$. The writers plan to return to this question.

\section{BIBLIOGRAPHY}

1. S. Bergman, The kernel function and conformal mapping, Mathematical Surveys, No. 5, New York, 1950.

2. J. A. Clarkson, Uniformly convex spaces, Trans. Amer. Math. Soc. vol. 40 (1936) pp. 396-414.

3. P. R. Garabedian, The classes $L_{p}$ and conformal mapping, Trans. Amer. Math. Soc. vol. 69 (1950) pp. 392-415. $371-402$.

4. H. Lebesgue, Sur le problème de Dirichlet, Rend. Circ. Mat. Palermo vol. 24 (1907) pp.

5. J. L. Walsh, Interpolation and approximation by rational functions in the complex domain, Amer. Math. Soc. Colloquium Publications, vol. 20, 1935.

6. - On interpolation and approximation by functions analytic and bounded in a given region, Proc. Nat. Acad. Sci. U.S.A. vol. 24 (1938) pp. 477-486.

7. - On interpolation by functions analytic and bounded in a given region, Trans. Amer. Math. Soc. vol. 46 (1939) pp. 46-65.

8. - Overconvergence, degree of convergence, and zeros of sequences of analytic functions, Duke Math. J. vol. 13 (1946) pp. 195-234.

9. - The location of critical points of analytic and harmonic functions, Amer. Math. Soc. Colloquium Publications, vol. 34, 1950. 
10. J. L. Walsh and Philip Davis, Interpolation and orthonormal systems, Journal d'Analyse Mathématique vol. 2 (1952) pp. 1-28.

11. J. L. Walsh and E. N. Nilson, On functions analytic in a region: approximation in the sense of least pth powers, Trans. Amer. Math. Soc. vol. 65 (1949) pp. 239-258.

12. J. L. Walsh, An interpolation problem for harmonic functions, Amer. J. Math. vol. 76 (1954) pp. 259-272.

13. J. L. Walsh and J. P. Evans, Note on the distribution of zeros of extremal polynomials, Proc. Nat. Acad. Sci. U.S.A. vol. 40 (1954) pp. 332-337.

Wellesley College,

Wellesley, Mass.

HARVARD UNIVERSITY,

Cambridge, Mass. 